\title{
Identification of positive and negative mediators of fluconazole tolerance in Candida albicans
}

$\underline{\text { E. Delarze }}^{1}$, C. Pralong ${ }^{1}$, F. Maranzano ${ }^{1}$, S. Znaidi' ${ }^{2}$, M. Legrand ${ }^{2}$, M. Arendrup ${ }^{3}$, C. Munro ${ }^{4}$, C. d'Enfert ${ }^{2}$, D. Sanglard ${ }^{1}$;

${ }^{1}$ University of Lausanne and University Hospital Center, Lausanne, SWITZERLAND, ${ }^{2}$ Pasteur Institute, Paris, FRANCE,

${ }^{3}$ Statens Serum Institut, Copenhagen, DENMARK, ${ }^{4}$ University of Aberdeen, Aberdeen, UNITED KINGDOM

\section{Candida albicans and tolerance}

- Problems of resistance to antifungal treatments

- Increased Minimum Inhibitory Concentration (MIC, first concentration inhibiting $\leq 50 \%$ of growth)

- Tolerance $\rightarrow$ ability to survive high drug concentrations without development of resistance mechanisms [1]

- Increased residual growth [1]

- Tolerance mainly observed for fluconazole (FLC), the most widely used antifungal

- Tolerance may play a role in treatment failure and disease persistence

- Mechanisms of tolerance are still poorly understood

Aims of the project

- Identification and characterization of mediators of tolerance to FLC

- Mediators of tolerance $\rightarrow$ new drug targets and treatment strategies improvement

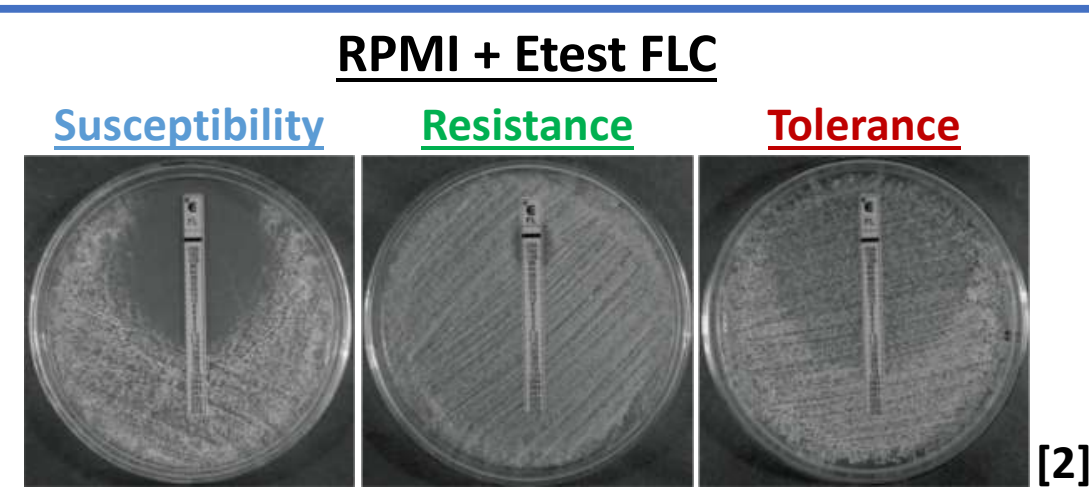

Resistance

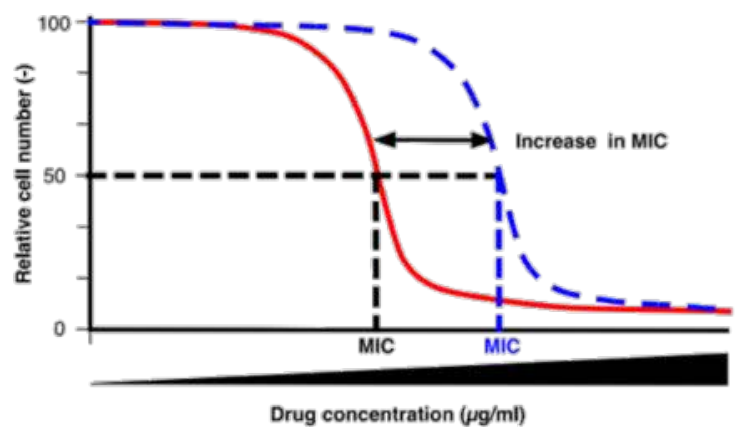

Tolerance

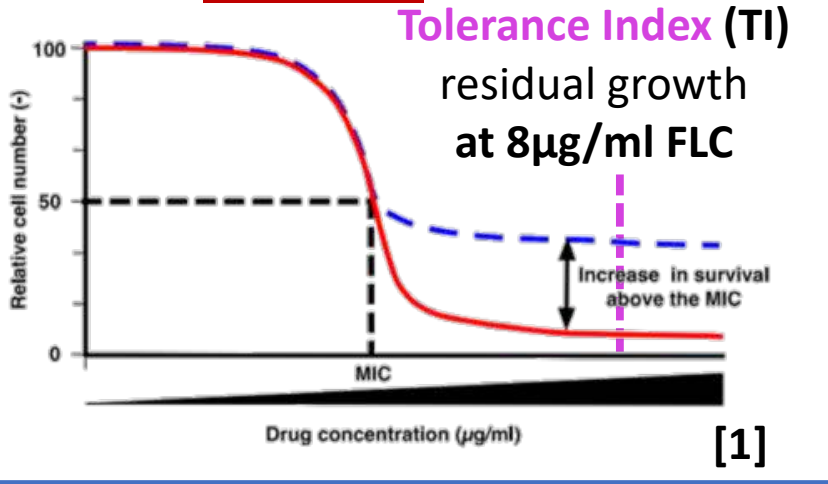

Identification of putative tolerance mediators

- Collection of $\mathbf{5 8 2}$ barcoded (BC) Doxycycline (Dox)-dependent overexpression (OE) C. albicans strains

- Collection constructed in C. albicans BWP17 genetic background

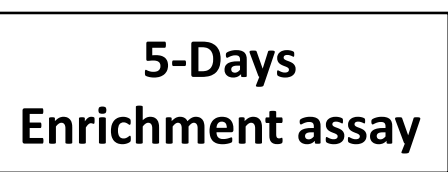

\begin{tabular}{|c|}
\hline DNA extraction and \\
BC amplification
\end{tabular}
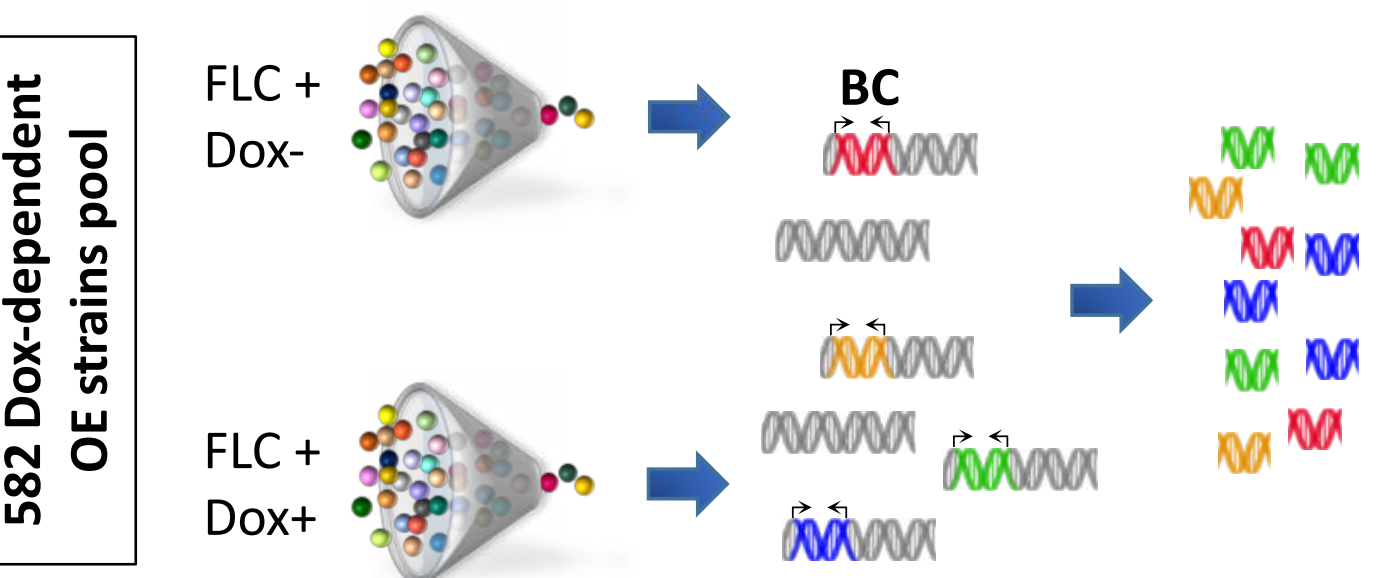

RPMI 2\% Glc $10 \mu \mathrm{g} / \mathrm{ml}$ Dox $10^{7}$ cells $/ \mathrm{ml}$ \begin{tabular}{l|c|c|c}
$\mathrm{pH} 6$ & $1 \mathrm{mM} \mathrm{FeCl} 3$ & $32 \mu \mathrm{g} / \mathrm{ml} \mathrm{FLC}$
\end{tabular}

- Collection pooled and maintained in presence or absence of strong FLC pressure $(32 \mu \mathrm{g} / \mathrm{ml})$ and Dox $(10 \mu \mathrm{g} / \mathrm{ml})$ to enrich in resistant and/or tolerant strains

- Selection of strains $\geq 2 x$ enriched (activators) and $\geq 2 x$ depleted (repressors) after 5 days of enrichment

- Selection with single strains (79 + 39 strains from pools) FLC susceptibility assays: 2fold increase/decrease with FLC and +/-DOX

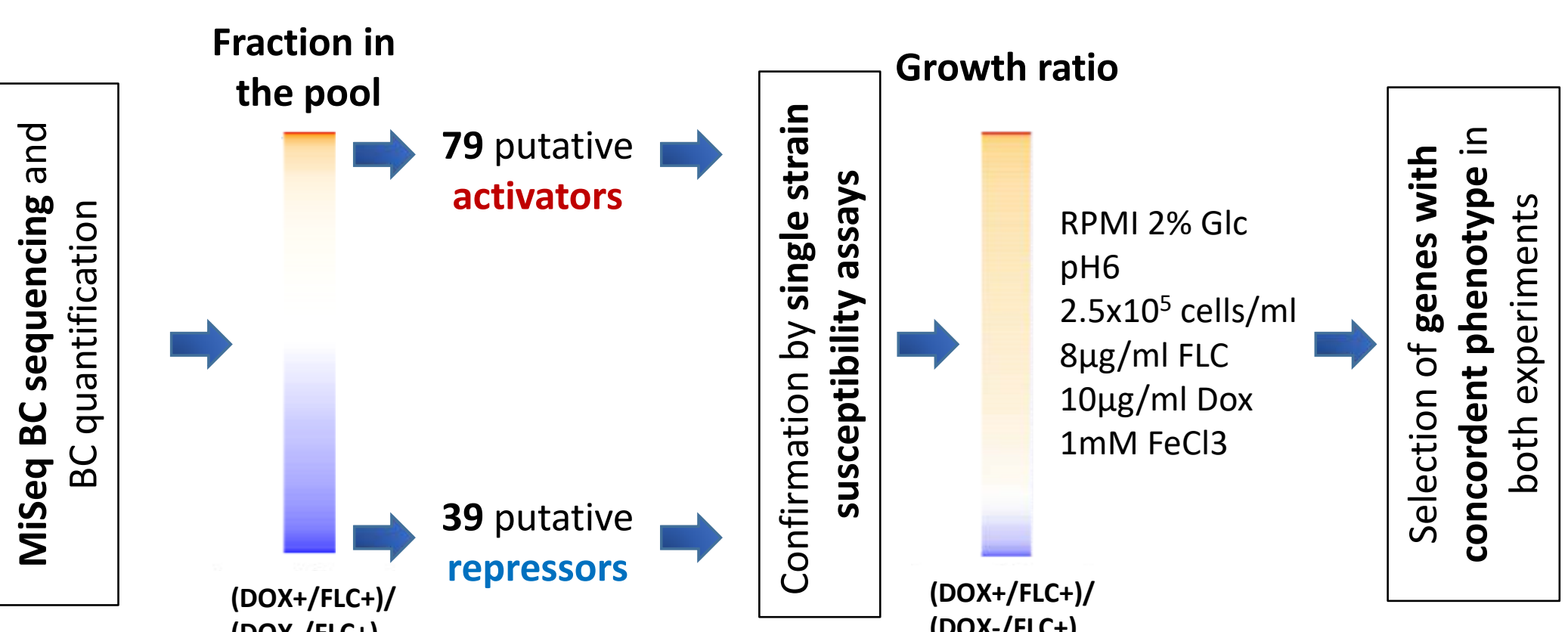

(DOX-/H (DOX-/FLC+)

Putative activators

Single strain FLC susceptibility assays

- Enrichment assay and individual strain susceptibility assay gave 12 putative activators and 15 putative repressors

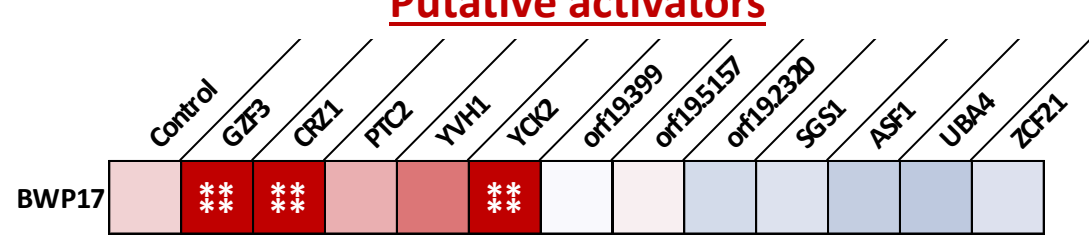

- Determination of the Dox+/Dox-growth ratios in presence of $8 \mu \mathrm{g} / \mathrm{ml} \mathrm{FLC} \mathrm{at} \mathrm{pH} 6$

- Since BWP17 basal tolerance was too low to detect reduction of tolerance, tolerant clinical isolates were used putative tolerance repressors

- DSY2110 ( $\mathrm{TI}=\mathbf{2 7 . 4 \%}$ of growth control)

- DSY4754 ( $\mathrm{TI}=68.5 \%$ of growth control)

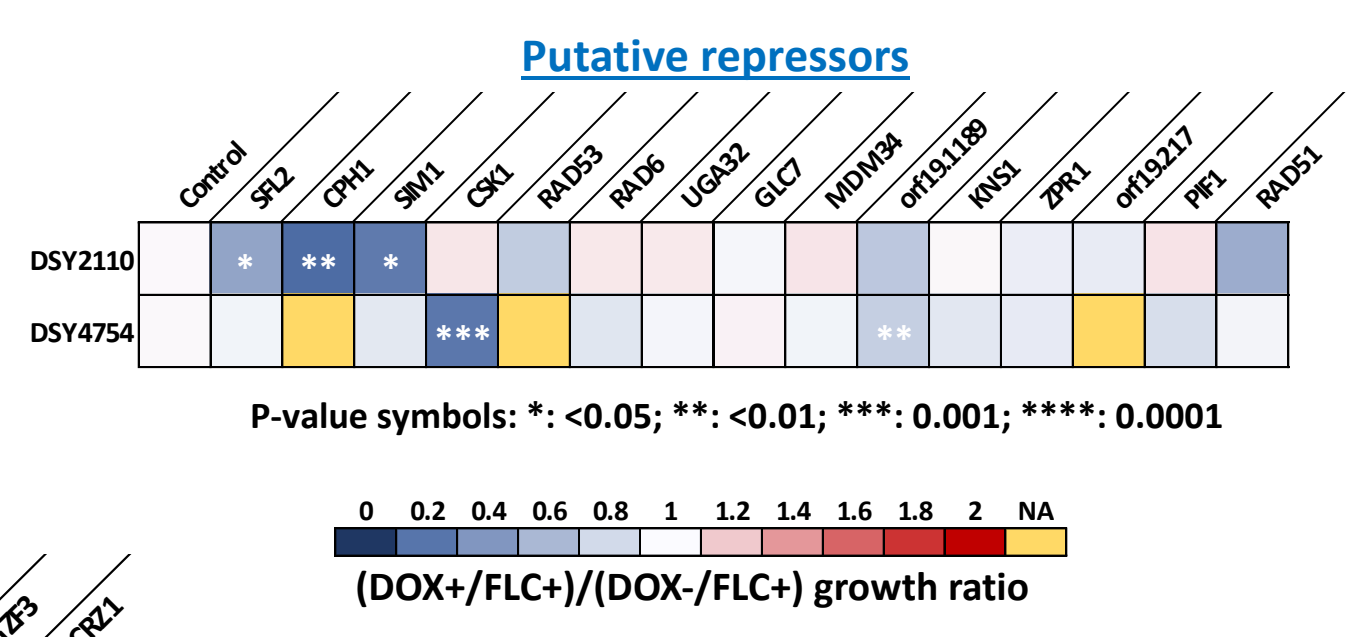

\begin{tabular}{c|c}
\hline Gene name & $\underline{\text { Function }}$ \\
\hline GZF3 & GATA-type transcription factor \\
\hline CRZ1 & $\begin{array}{c}\text { Calcineurin-regulated C2H2 } \\
\text { transcription factor }\end{array}$ \\
\hline YCK2 & $\begin{array}{c}\text { Plasma membrane protein similar to } \\
\text { S. cerevisiae casein kinase I }\end{array}$ \\
\hline
\end{tabular}

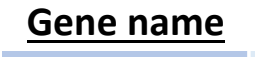

CSK1

CPH1

SFL2
Function

Putative mitogen-activated protein (MAP) kinase with an unknown role Transcription factor; for mating, filamentation on solid, pheromonestimulated biofilms

Transcription factor involved in regulation of morphogenesis
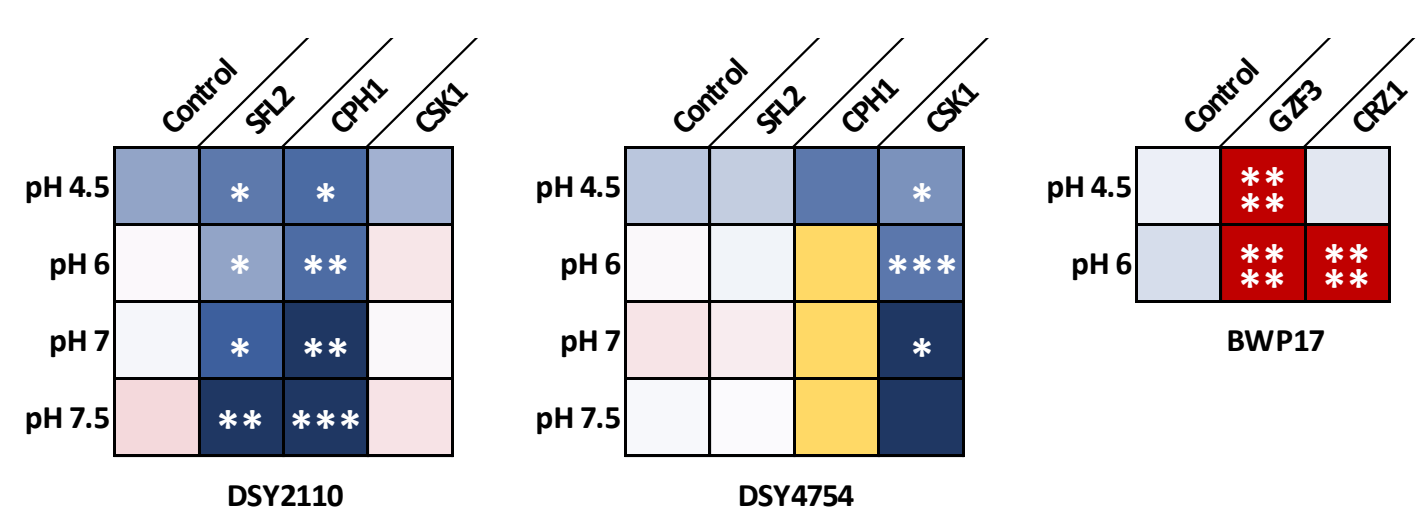

- CRZ1, GZF3, CSK1, CPH1 and SFL2 showed consistent increase/decrease of tolerance when $\mathrm{OE}$ independently of the $\mathrm{pH}$ tested

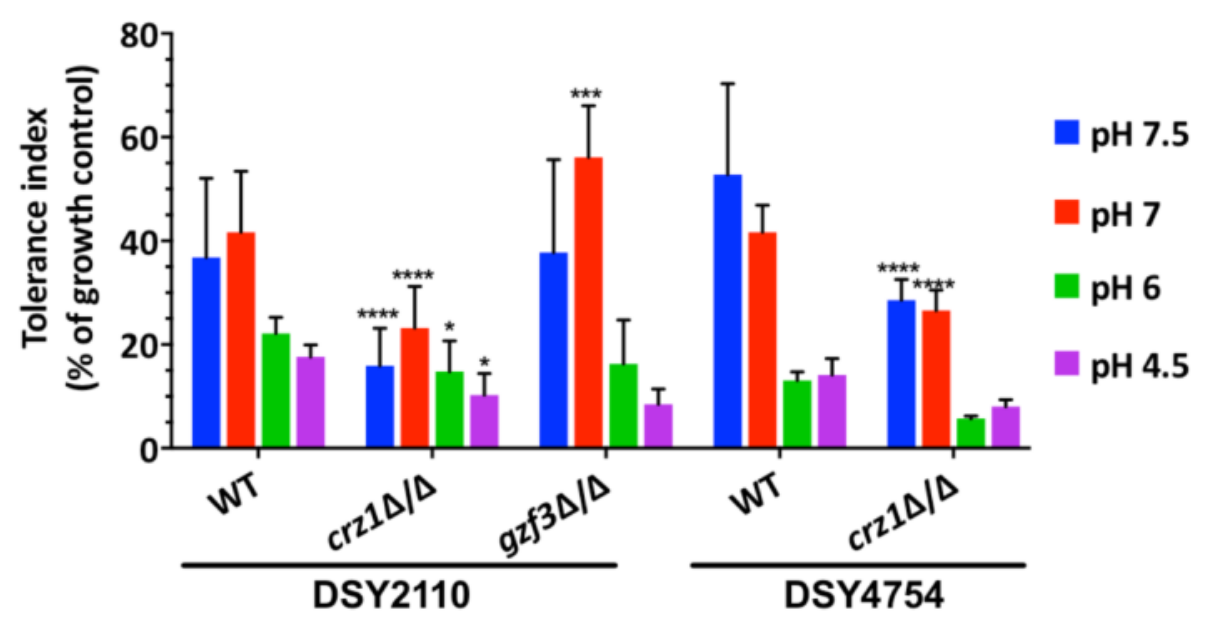

- Inactivation of CRZ1 in clinical strains decreases tolerance. Inactivation of GZF3 has no significant impact on tolerance in these strains.

\section{Conclusions}

- The Dox-dependent OE system and our enrichment strategy allowed us to identify 2 potential activators and 3 potential repressors of FLC tolerance

- CRZ1 and GZF3 were observed to increase the residual growth by 2fold in BWP17 when OE at pH6.

- Overexpression of YCK2 did not reach our tolerance cutoff $(\mathrm{TI} \geq 0.2)$

- Overexpression of CSK1 reduced tolerance by 5-fold (in DSY4754), while CPH1 and SFL2 yielded 6- and 2-fold tolerance reduction (in DSY2110).

- The deletion of $C R Z 1$ in clinical strains with high tolerance profiles reduces tolerance. 